\title{
Persistent Current Switch for HTS Superconducting Magnets: Design, Control Strategy and Test Results
}

\author{
Chao Li, Student Member, IEEE, Jianzhao Geng, Boyang Shen, Xinru Li, Jamie Gawith, Jun Ma, Jiabin Yang, \\ T. A. Coombs, Senior Member, IEEE
}

\begin{abstract}
High temperature superconductor (HTS) is becoming more and more attractive for the application in high field magnets which can be utilized in the magnetic resonance imaging (MRI) and rotating machines. To charge the HTS magnets and operate them in persistent current mode, a persistent current switch (PCS) is very necessary. This paper will focus on how to build a fast persistent current switch controlled by AC magnetic field. The PCS can present a large resistance rapidly compared with conventional PCS and recover to zero-resistance in a short recovery time. Impacting factors and strategy to control the proposed PCS are discussed. An experimental system is built and tests are carried out to verify the practicality of the proposed PCS.
\end{abstract}

Index Terms-Persistent current switch, dynamic resistance, uniform magnetic field, HTS tape.

\section{INTRODUCTION}

Superconductors are ideal materials for high field magnets, owing to their capability of transporting high-density DC with little power loss. With the development in manufacturing high temperature superconducting (HTS) tapes, HTS magnets are being developed in the application such as magnetic resonance imaging (MRI), and motor winding. HTS magnets can be driven directly by an external power source (a continuously driven mode) or can operate in the persistent current mode.

HTS magnets operating in the persistent current mode are able to maintain steady magnetic field and avoid the complex system and heat loss caused by operating in a continuously driven mode [1]. The key part enabling HTS magnets to operate in persistent current mode is the persistent current switch (PCS). Actually, the PCS can be regarded as an electrical component with variable resistance. The PCS is usually connected in parallel with the external dc power supply and the magnet coils. The PCS is required to present large resistance during the charging process so that the external dc power supply can energize the superconducting magnet. After energizing the magnet coils, the PCS can keep the magnets coils operating in persistent mode through returning to zero-resistant state to short-circuit the external dc power supply. Therefore, the PCS should possess the following features: (a) zero-resistance in persistent mode; (b) large resistance during the charging process; (c) quick transition between the above two states.

This work was supported by the CSC Cambridge Trust. (Corresponding author: Jianzhao Geng)

The authors are with Electrical Engineering Division, Department of Engineering, University of Cambridge, Cambridge CB3 0FA, UK (e-mail: jg717@cam.ac.uk).
The PCS usually achieve above features through the transitions of the superconductor between the superconducting state (zero-resistance state) and the normal state. Thermally-controlled switches trigger the transition though heating the superconductor above its critical temperature [2-6]. This type of PCS can achieve a high resistance, but it usually takes several seconds or even longer to transfer between the two states. [7] proposed a fast switch controlled by field, whose respond and recovery is very short, but the resistance it can present is too small. In some circumstance such as flux pumping, it is necessary to develop a fast PCS with high resistance.

In this paper, we report a design and experimental results of a PCS, controlled by alternating magnetic field at normal frequency which is generated by copper coils wounded around the iron core. The proposed PCS can achieve high resistance efficiently and requires little response time and recovery time.

\section{Structure Design AND Control StRAtegy of THE PERSISTENT CURRENT SWITCH}

\section{A. Structure Design of PCS}

The PCS mainly consists of three parts: the iron cores, copper coils, and a superconductor tape, as shown in Fig.1.

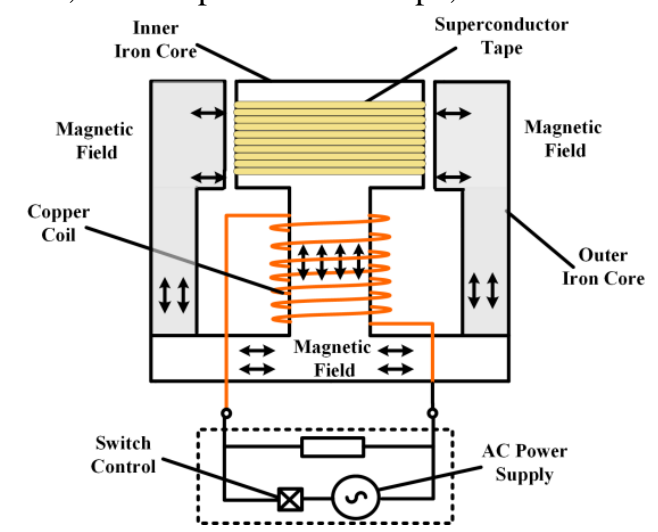

Fig. 1. Schematic diagram of the PCS[8]

The iron cores, composed of the inner iron core and the outer iron core, serve as the path for the magnetic field and hold the superconducting tape and a copper coil. The copper coil, controlled by external ac power supply, can generate homogeneous magnetic field applied perpendicular to the face of the superconducting tape.

The superconducting tape, wound around the inner iron core, is connected to the superconducting magnet it energizes. To avoid the induced voltage from the copper coil, the superconducting tape should be wound in the bifilar manner[8]. Two superconducting tapes are soldered together at one end by the same soldering technique used in $[1,9,10]$. 
In this method, the current flowing in tape I and tape II will be the equal in magnitude and opposite in direction. Hence, the induced voltage from the copper coil will be canceled.

\section{B. Working Principle of the PCS}

A response voltage, called the dynamic-resistance voltage, is induced if a superconducting tape carrying dc current under external applied ac magnetic field. This is caused by net flux motion from one side of the superconductor to the other. And this can be regarded as redistributing the vortices inside the sample. The response voltage will enter another stage, namely flux flow voltage, if the superconducting tape is further driven into the flux flow regime, where small changes in the pinning energy largely affect the flux motion [11-15]. The PCS is able to charge the superconducting magnet through taking advantage of the response voltage of current-carrying superconducting tape under ac magnetic field.

A calculation method of the dynamic-resistance was proposed by Oomen as follows [16]:

$$
R_{d y n}=\frac{4 a l f}{I_{c 0}}\left(B_{a}-B_{a, t h}\right)
$$

Where $a$ is the half width of the superconductor slab, $l$ is the length of the superconductor slab, $f$ is the frequency of the applied ac magnetic field, $B_{a}$ is the amplitude of the applied field and $B_{a, t h}$ is the threshold of the applied field that could totally penetrate the superconductor.

If the transport current is smaller than the critical current, no quench will occur in the superconducting tape during the whole process. As a consequence, the dynamic resistance will be able to disappear nearly immediately after the applied alternating magnetic field is removed. According to whether the dynamic resistance appears or not, the working state of the PCS can be classed into two states, "persistent current mode" state and "charging" state. The working state can be switched through controlling the current in the copper coil. If no current is transporting in the copper coil, no ac magnetic field will be applied to the superconducting tape of the PCS. According to Eq.1, the value of the dynamic resistance $R_{d y n}$ is zero. This scenario corresponds to the "persistent current mode". If ac current is applied in the copper coil, ac magnetic field will be generated perpendicular to the superconducting tape. Large dynamic resistance appears to charge the superconducting magnet and the working state of the PCS is called the "charging mode".

\section{Control Strategy of the PCS}

Fig. 2 shows the schematic diagram of how the PCS can be controlled to charge the superconducting magnet. The whole process of charging the superconducting magnet can be divided into four steps: 1. External DC power supply is turned on; 2. AC current is applied in the coper coil to generate ac field to switch the PCS into "charging mode"; 3. AC current in the copper coil is cut off to switch the PCS into "persistent current mode"; 4. External DC power supply is off to stop the whole charging process. The charging speed of the superconducting magnet depends on the dynamic resistance presented by the PCS. And the dynamic resistance can be altered through controlling the frequency and amplitude of the applied AC magnetic field. Hence, it will be more flexible and efficiently to charge the superconducting magnet, utilizing the proposed PCS.

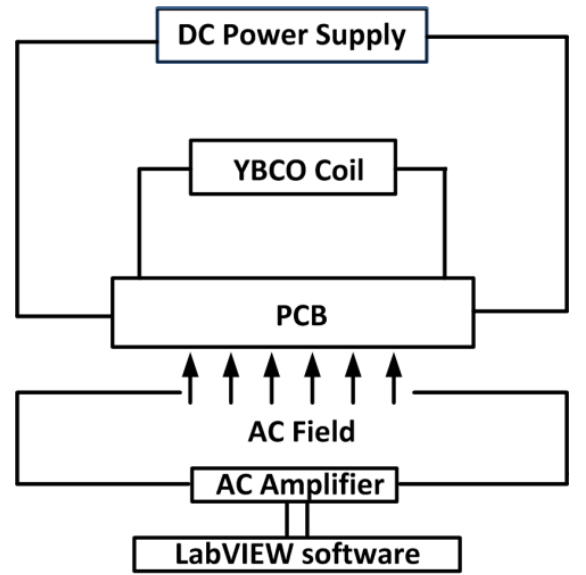

Fig. 2. Schematic diagram of the Persistent Current Switch for charging the superconducting magnet.

\section{EXPERIMENTAL TESTS AND DISCUSSION}

\section{A. Experimental System}

Experiments and measurements have been carried out in the real system to test the performance of the proposed PCS, as shown in Fig.3.

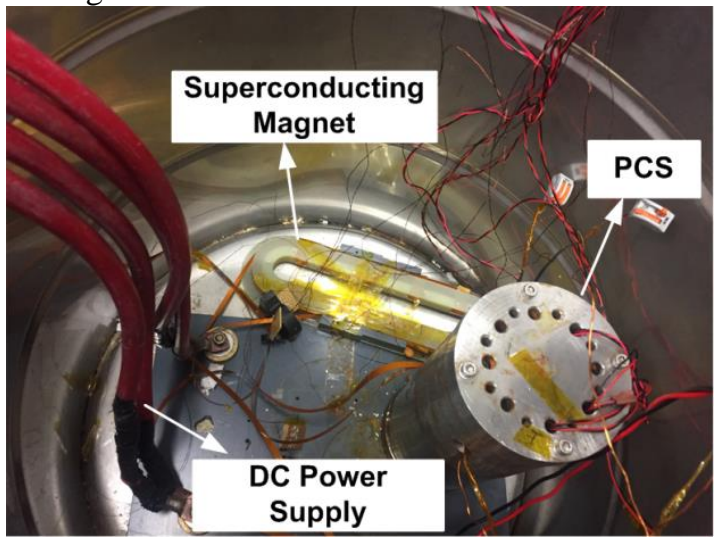

Fig. 3. Photograph of the experimental system.

The superconducting tape wound around the inner iron core of the PCS was a $60 \mathrm{~cm}$-long YBCO tape with the critical current of $80 \mathrm{~A}$. The copper coil was powered by the Europower amplifier which was controlled by a LabVIEW program. The load current $i_{L}$ was measured by a Hall sensor fixed in the center of the double pancake superconducting coil. A NI-PCIe 6221 DAQ card was used to acquire the digital signal, with a $20 \mathrm{kHz}$ sampling rate. A double-pancake coil was fabricated with a SuperPower YBCO tape that has a dimension of $4 \mathrm{~mm}$ width and $0.1 \mathrm{~mm}$ thickness. The inductance of the double-pancake coil is $2.56 \mathrm{mH}$. The double-pancake coil served as the superconducting magnet which was going to be charged and its critical current was 37A. The whole system was cooled in the liquid nitrogen.

\section{B. Performance Test}

In order to charge the superconducting coil to its critical current, the charging current was set to be $45 \mathrm{~A}$, which is slightly larger than the critical current of the superconducting 
coil. The magnitude of the applied AC field is $100 \mathrm{mT}$, and the frequency of the applied AC field is chosen to be $50 \mathrm{~Hz}$. Fig.4 shows the load current curve of the superconducting coil during the charging process. It can be observed that the load current of the superconducting coil increases a little as soon as the DC power supply is turned on in spite of no applied AC magnetic field. This can be attributed to the soldering joint resistance in the PCS, which generates a low voltage to charge the superconducting coil a little bit. The charging process actually begins when AC magnetic field is applied to switch off the PCS. Correspondingly, large DC voltage occurs across the PCS and the load current of the superconducting magnet starts to increase rapidly to its critical current 37A. It should be noted that the response time of the PCS is less than ten milliseconds.

After the charging process is completed, the superconducting coil is able to step into the persistent current mode immediately both the AC magnetic field and the DC power supply are removed. This can demonstrate that it takes little time for the resistance of the PCS to recover to zero. Hence, it will be more efficient to charge the superconducting coil, utilizing the proposed PCS considering its flexibility in the control of the PCS resistance.

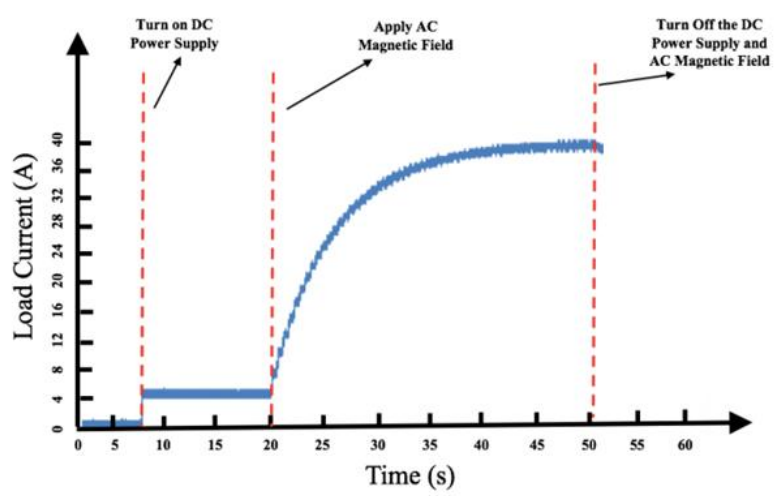

Fig. 4. Load current curve of the superconducting magnet. The frequency of the applied ac field is $50 \mathrm{~Hz}$ and the amplitude of the applied ac field is $100 \mathrm{mT}$.

After charging the superconducting coil to around $40 \mathrm{~A}$, some load current decay of the superconducting magnet can also be observed in the Fig.4. This fast current decay can be mainly attributed to the flux flow resistance of the coil superconductor and the soldering joint resistance in the whole experimental system. With the rapid development in the superconducting joints, the decay of the load current will be significantly improved.

\section{Influence of the Field Magnitude and Frequency}

In order to effectively control the performance of the PCS, we have to investigate two factors, namely the field magnitude and field frequency. Fig.5 shows the resistance curve of the PCS under different field magnitudes. It can be easily observed that the resistance of the PCS grows with the increase of the field magnitude. At the stage when the field magnitude is relatively small (say less than $40 \mathrm{mT}$ ), the resistance across the PCS is nearly zero. This is because the magnitude of the applied field has to be larger than that of the threshold field, to totally penetrate the superconductor, mentioned in Equ.1. When the field magnitude exceeds the threshold, the PCS will present resistance.

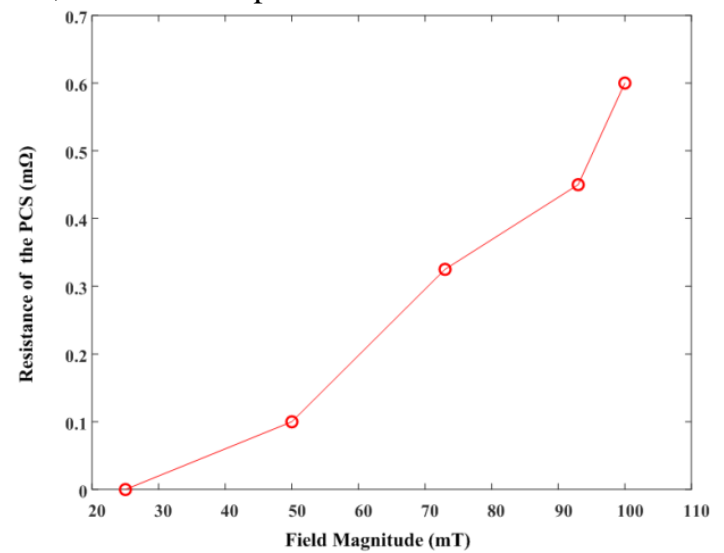

Fig. 5. Load current curve of the superconducting magnet. The frequency of the applied ac field is $50 \mathrm{~Hz}$ and the amplitude of the applied ac field is $100 \mathrm{mT}$

The frequency of the applied magnetic field can also impact the performance of the PCS. In this test, the charging current from the DC power supply was set to be 35 A. Fig. 6 shows the curve of the current of the superconducting coil under the magnetic field with different frequency. The magnitude of the magnetic field is $65 \mathrm{mT}$. The red curve, blue curve and green curve correspond to $200 \mathrm{~Hz}, 100 \mathrm{~Hz}$, and $50 \mathrm{~Hz}$ respectively. The charging process is triggered nearly immediately when the magnetic field starts to be applied in the PCS. It can be observed that the charging speed is positively related to the frequency. This is because high frequency of the magnetic field will induce large dynamic-resistance. And the larger the dynamic-resistance is, the larger the voltage across the PCS will be charge the superconducting coil. It should be noted that the difference of the final superconducting coil current results from the soldering resistance between the PCS and the superconducting coil. The voltage across the PCS is equal to the total of the voltage of the soldering-resistance and the voltage across the superconducting coil. Therefore, the larger dynamic-resistance voltage, the final current of the superconducting coil will achieve.

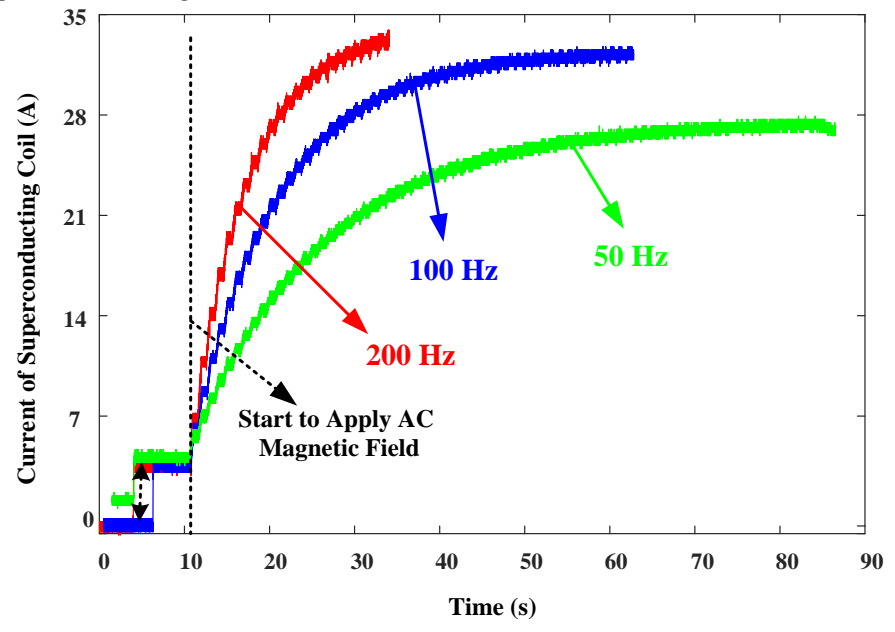

Fig. 6. Curve of the current of the superconducting coil under $60 \mathrm{mT}$ ac magnetic field with different frequency. 


\section{Discussion}

In the thermally-controlled PCS, electrical heaters are required to drive the PCS to the normal state to charge the superconducting magnet. After the completion of the charging process, the persistent current mode will not start until the PCS recovers to the superconducting state from the normal state. Hence, some techniques have to be applied to accelerate the recovery of the PCS, otherwise the load current of the superconducting magnet will be consumed by the resistance of the PCS. It usually takes several seconds for the conventional thermally-controlled PCS to respond or recover. And large amount of loss will be generated in the whole process. Owing to its fast response and recovery, the proposed PCS can solve above problems. Besides, the value of the resistance of the PCS can be flexibly controlled through adjusting the frequency and the magnitude of the applied field. Considering the switching off resistance, response speed and flexibility, the proposed PCS has promising potential to be utilized in charging high temperature superconducting magnets[17].

The structure of the PCS makes it possible to utilize long superconductor tape, which could make its resistance much larger. Hence, the superconducting fault current limiter[18, 19] can be built in the form of the proposed PCS. And the applied field by the copper coil can help to accelerate the quench process the superconducting tape.

\section{CONCLUSION}

A field-controlled PCS for HTS magnets, wound from YBCO tape, immersed in liquid nitrogen is developed and tested. Different from the conventional PCS forcing the superconductor to the normal state through heating, the proposed PCS is taking advantage of the dynamic resistance and flux flow resistance which origin from the external AC magnetic field. The advantage of the field-controlled PCS is its flexibility in controlling the value of the switching off resistance. The response and recovery time of the PCS is less than 10 milliseconds. As long as the superconducting tape of the proposed PCS is long enough, the structure design can make it possible that the switch-off resistance is large enough for charging a high-filed magnet rapidly. Hence, the proposed PCS is very promising in the application of charging the superconducting magnet.

\section{ACKNOWLEDGEMENT}

The authors would like to acknowledge Mr J. Grundy for his help in setting up the experiment. C. Li would like to acknowledge Cambridge Trust for offering the Cambridge CSC Scholarship (No. 201506250125) to support his study in Cambridge.

\section{REFERENCES}

[1] T. Qu, P. C. Michael, J. Voccio, J. Bascunán, S. Hahn, and Y. Iwasa, "Persistent-current switch for pancake coils of rare earth-barium-copper-oxide high-temperature superconductor: Design and test results of a double-pancake coil operated in liquid nitrogen
(77-65 K) and in solid nitrogen (60-57 K)," Applied physics letters, vol. 109, no. 8, pp. 082601, 2016.

[2] T. Tosaka, T. Kuriyama, M. Yamaji, K. Kuwano, M. Igarashi, and M. Terai, "Development of a persistent current switch for HTS magnets," IEEE transactions on applied superconductivity, vol. 14, no. 2, pp. 1218-1221, 2004.

[3] C. Cui, J. Cheng, S. Chen, L. Li, and X. Hu, "Design and Test of Superconducting Persistent Current Switch for Experimental Nb $3 \mathrm{Sn}$ Superconducting Magnet," IEEE Transactions on Applied Superconductivity, vol. 26, no. 4, pp. 1-4, 2016.

[4] P. C. Michael, T. Qu, J. Voccio, J. Bascuñán, S. Hahn, and Y. Iwasa, “A REBCO persistent-current switch (PCS): Test results and switch heater performance," IEEE Transactions on Applied Superconductivity, vol. 27, no. 4, pp. 1-5, 2017.

[5] K. Takahashi, T. Hase, S. Awaji, A. Nakai, S. Yamano, S. Mukoyama, and H. Sakamoto, "Performance of an HTS Persistent Current System for REBCO Pancake Coil," IEEE Transactions on Applied Superconductivity, vol. 28, no. 3, pp. 1-4, 2018.

[6] P. C. Michael, J. Lee, J. Voccio, J. Bascuñán, S. Hahn, and Y. Iwasa, “A REBCO Persistent-Current Switch, Immersed in Solid Nitrogen, Operating at Temperatures Near $10 \mathrm{~K}$," IEEE Transactions on Applied Superconductivity, vol. 28, no. 3, pp. 1-5, 2018.

[7] J. Geng, K. Matsuda, B. Shen, H. Zhang, X. Zhang, L. Fu, Z. Huang, and T. Coombs, "HTS persistent current switch controlled by AC magnetic field," IEEE Transactions on Applied Superconductivity, vol. 26, no. 3, pp. 1-4, 2016.

[8] C. Li, J. Geng, J. Gawith, B. Shen, X. Zhang, H. Zhang, J. Ma, and T. A. Coombs, "Design for A Persistent Current Switch Controlled by Alternating Current Magnetic Field," IEEE Transactions on Applied Superconductivity, 2018.

[9] S. Liu, X. Jiang, G. Chai, and J. Chen, "Superconducting joint and persistent current switch for a 7-T animal MRI magnet," IEEE Transactions on Applied Superconductivity, vol. 23, no. 3, pp. 4400504-4400504, 2013.

[10] T. Lécrevisse, J. Bascuñán, S. Hahn, Y. Kim, J. Song, and Y. Iwasa, "Tape-to-tape joint resistances of a magnet assembled from (RE) BCO double-pancake coils," IEEE Transactions on Applied Superconductivity, vol. 25, no. 3, pp. 1-5, 2015.

[11] E. H. Brandt, and G. P. Mikitik, "Why an ac magnetic field shifts the irreversibility line in type-II superconductors," Physical review letters, vol. 89, no. 2, pp. 027002, 2002.

[12] Z. Jiang, R. Toyomoto, N. Amemiya, X. Zhang, and C. W. Bumby, "Dynamic resistance of a high-T c coated conductor wire in a perpendicular magnetic field at $77 \mathrm{~K}$," Superconductor Science and Technology, vol. 30, no. 3, pp. 03LT01, 2017.

[13] Z. Jiang, W. Zhou, Q. Li, M. Yao, J. Fang, N. Amemiya, and C. W. Bumby, "The dynamic resistance of YBCO coated conductor wire: Effect of DC current magnitude and applied field orientation," Superconductor Science and Technology, vol. 31, no. 3, pp. 035002 , 2018.

[14] A. Uksusman, Y. Wolfus, A. Friedman, A. Shaulov, and Y. Yeshurun, "Voltage response of current carrying $\mathrm{Y}-\mathrm{Ba}-\mathrm{Cu}-\mathrm{O}$ tapes to alternating magnetic fields," Journal of Applied Physics, vol. 105, no. 9, pp. 093921, 2009.

[15] J. Geng, C. Li, and T. A. Coombs, “A Fast AC Field Controlled Impedance in HTS Coated Conductors: Response Speed and Electric Field Value," IEEE Transactions on Applied Superconductivity, vol. 27, no. 6, pp. 1-5, 2017.

[16] M. Oomen, J. Rieger, M. Leghissa, B. ten Haken, and H. H. ten Kate, "Dynamic resistance in a slab-like superconductor with Jc (B) dependence," Superconductor science and technology, vol. 12, no. 6, pp. 382, 1999.

[17] J. Geng, and T. Coombs, "Mechanism of a high-Tc superconducting flux pump: Using alternating magnetic field to trigger flux flow," Applied Physics Letters, vol. 107, no. 14, pp. 142601, 2015.

[18] B. Li, C. Li, F. Guo, and Y. Xin, "Overcurrent protection coordination in a power distribution network with the active superconductive fault current limiter," IEEE Transactions on Applied Superconductivity, vol. 24, no. 5, pp. 1-4, 2014.

[19] B. Li, C. Li, and F. Guo, "Application studies on the active SISFCL in electric transmission system and its impact on line distance protection," IEEE Transactions on A Superconductivity, vol. 25, no. 2, pp. 1-9, 2015. 